\title{
Aminoguanidine Treatment Increases Elasticity and Decreases Fluid Filtration of Large Arteries from Diabetic Rats
}

\author{
Maya S. P. Huijberts, * Bruce H. R. Wolffenbuttel, * Harry A. J. Struijker Boudier, ${ }^{*}$ Francy R. L. Crijns, * \\ Arie C. Nieuwenhuijzen Kruseman, * Pierre Poitevin, ${ }^{3}$ and Bernard I. Lévy \\ ${ }^{*}$ Division of Endocrinology \& Metabolism, Department of Internal Medicine, University Hospital Maastricht; ${ }^{\ddagger}$ Department of \\ Pharmacology, University of Limburg, Maastricht, The Netherlands; and ${ }^{\S}$ Institut National de la Santé \\ et de la Recherche Medicale Unité 141, Paris, France
}

\begin{abstract}
The accumulation of advanced glycosylation endproducts (AGEs) on collagen and the subsequent stiffening of this matrix protein in diabetes has been described many years ago. Structural modification of collagen in the arterial wall might have important effects on arterial elasticity. Aminoguanidine is known to decrease the formation of AGEs. In this study we evaluated the effects of aminoguanidine treatment on different parameters reflecting arterial wall elasticity in diabetic rats. We demonstrated that treatment of diabetic rats with aminoguanidine resulted in a significant increase in carotid static compliance ( $+39 \%, P<0.01$ under control conditions, and $+27 \%, P$ $<0.01$ after abolition of vascular tone by $\mathrm{KCN}$ ), and a decrease in characteristic aortic input impedance $(-40 \%, P<0.01)$. The arterial pulse pressure in aminoguanidine-treated rats was decreased $(-15 \%, P<0.05)$ and the pulsatile component of left ventricular power output was relatively diminished $(-35 \%, P$ $<0.05$ ). In addition, we observed a lower fluid filtration across the carotid wall. These results indicate an increased vascular elasticity, an improved left ventricular-arterial coupling, and a decreased vascular permeability in diabetic rats after aminoguanidine treatment, suggesting that AGE-accumulation on collagen negatively affects arterial wall properties in experimental diabetes. (J. Clin. Invest. 1993. 92:1407-1411.) Key words: diabetic angiopathies • glycosylation • carotid arteries • compliance $\bullet$ vascular permeability
\end{abstract}

\section{Introduction}

There is growing evidence that the formation of so-called advanced glycosylation endproducts (AGEs), is involved in the development of diabetic angiopathy, which remains one of the most severe complications of diabetes mellitus. AGEs are the final product of a series of complex rearrangements resulting from the reaction of circulating sugars with proteins. The formation of these products is irreversible, leading to accumula-

Address correspondence to Dr. Maya S. P. Huijberts, Division of Endocrinology \& Metabolism, Department of Internal Medicine, University Hospital Maastricht, P.O. Box 5800, 6202 AZ Maastricht, The Netherlands.

Received for publication 25 February 1993.

1. Abbreviations used in this paper: AGE, advanced glycosylation endproduct.

J. Clin. Invest.

(c) The American Society for Clinical Investigation, Inc.

0021-9738/93/09/1407/05 \$2.00

Volume 92, September 1993, 1407-1411 tion of AGEs, especially on vascular wall proteins with a slow turnover rate. One of the firstly recognized features of AGEformation is the accumulation of AGEs on collagen (1-3), forming intermolecular bonds leading to an increased stiffness of collagen fibers. The effects of AGE-accumulation on collagen fibers for the mechanical properties of the arterial wall are not completely known. Because large compliance arteries are not only conduit vessels but also play a major role in the transmission and damping of the pulse wave along the systemic arterial tree, the mechanical properties of the arterial wall are major determinants of the left ventricular-arterial coupling. Therefore, alterations of mechanical properties of the arterial wall might have important consequences on cardiovascular performance in diabetes. Aminoguanidine has been shown to block AGE-formation in vivo and in vitro $(4,5)$, primarily through reaction with Amadori-derived fragmentation products in solution (6).

The aim of the present study was to evaluate the effects of long-term treatment with aminoguanidine on the mechanical properties of large compliance arteries in experimental diabetes. We demonstrated that large arterial elasticity was significantly increased by aminoguanidine treatment, leading to a decreased pulse pressure and a decreased left ventricular wt to body wt index. In addition, fluid filtration across the carotid arterial wall was significantly reduced. Therefore, our results suggest that aminoguanidine improved mechanical arterial wall properties and cardiac load in diabetic rats: the increase of vascular elasticity and the reduced permeability of the vascular wall after aminoguanidine treatment might protect the vascular system in (experimental) diabetes.

\section{Methods}

Animals. Male Wistar Rp rats were provided by TNO-REPGO, Rijswijk, The Netherlands. Diabetes was induced in all rats at the age of 10 wk by intraperitoneal injection with $70 \mathrm{mg} / \mathrm{kg}$ streptozotocin in citrate buffer, $\mathrm{pH}$ 4.5. After confirmation of the development of hyperglycemia ( $2 \mathrm{~d}$ later) by blood glucose determination using a hexokinase method, rats were randomized into a vehicle-treated diabetic group ( $n$ $=9)$, and a treatment group $(n=10)$ receiving daily injections with 50 $\mathrm{mg} / \mathrm{kg}$ aminoguanidine hemisulphate (Sigma Chemical Co., St. Louis, $\mathrm{MO}$ ) in PBS.

Determination of blood pressure, characteristic aortic input impedance, and left ventricular power output. Rats were studied 10-12 wk after induction of diabetes. The surgical procedure and hemodynamic measurements have been described in detail elsewhere (7). In summary, anesthesia was induced with $50 \mathrm{mg} / \mathrm{kg}$ i.p. pentobarbital. Rats were placed on a thermoregulated heating pad, intubated, and ventilated with a rodent respirator. A Teflon catheter $(0.9 \mathrm{~mm}$ i.d.) filled with saline, and coupled to a pressure transducer (model P23ID; Statham-Gould, Inc., Medical Products Div. Oxnard, CA) was inserted through the right carotid artery and placed into the ascending aorta. 
The catheter manometer system was checked for time and frequency, and showed a flat response beyond $60 \mathrm{~Hz}$. After performing a midsternal thoracotomy the ascending aorta was dissected free, and an adapted Doppler probe was positioned around the vessel to measure mean (cardiac output minus coronary blood flow) and phasic aortic blood flow. Following stabilization for $10 \mathrm{~min}$, aortic blood flow and pressure were recorded and processed by a microcomputer system (model Vectra 2821; Hewlett-Packard Co. Palo Alto, CA) with analog-digital converter (model Metrabyte; Data Translation Corp., Marlboro, MA). All parameters were calculated on a beat to beat basis for $30 \mathrm{~s}$ and then averaged.

The aortic input impedance spectrum was computed using Fourier analysis of the phasic aortic pressure and flow waves. Characteristic aortic input impedance was taken as the average value of the modulus of impedance for high frequencies (4th to 10th harmonic).

Total external ventricular work per unit time can be divided into two components. The first component, "steady flow power," $\dot{\mathrm{W}}_{\mathbf{s}}$, is determined mainly by the vascular resistance, and can be calculated as the product of mean pressure and mean flow. The second component, "oscillatory power," $\dot{\mathrm{W}}_{\mathbf{o}}$, is determined primarily by the dimensions and elasticity of the aorta and its side branches, and can be computed by multiplying the Fourier series expressions for pressure and flow. Total external left ventricular power output, $\dot{W}_{t}$, is the sum of $\dot{W}_{s}$ and $\dot{W}_{0}$. The ratio $\dot{W}_{0} / \dot{W}_{t}$ reflects the relative energy involved in pulsatile wave forms determined by characteristic aortic input impedance (8).

Determination of carotid artery compliance. After completion of the hemodynamic study, which never lasted more than $\mathbf{3 0}$ min, static mechanical properties of the carotid artery were measured. The distal end of the left carotid artery was dissected and cannulated with the tip of a nondistensible nylon catheter (length $10 \mathrm{~cm}, 0.6 \mathrm{~mm}$ i.d.), filled with a Tyrode's solution containing $4 \%$ albumin and $0.03 \%$ Evans blue. Albumin was added to the solution to preserve the endothelium (9) and to maintain a physiological osmotic gradient across the arterial wall. The tube was connected via a three-way connector $(a)$ to a pressure transducer (model P50; Gould Inc., Instruments Div., Cleveland, $\mathrm{OH}$ ), and $(b)$ to a syringe mounted on a computer controlled automatic pump (model 760; Harvard Apparatus, Inc., South Natick, MA). The root of the carotid artery was then dissected and a removable clamp was positioned at the junction of the carotid artery and the aortic arch. This preparation allowed us to isolate, in situ, $18-25 \mathrm{~mm}$ of nonexposed carotid (Fig. $1 \mathrm{a}$ ). The pressure in the artery was controlled by negative feedback. The arterial pressure was measured using the pressure transducer, and digitized using the analog-digital converter board and the microcomputer system. A BASIC program was developed to compare the measured pressure, $P_{\text {meas }}$, frequently ( 3 times per $s$ ) to a user-specified reference pressure, $P_{\text {ref }}$, and to command the computer-controlled pump to adjust the flow rate of fluid into the artery until $P_{\text {ref }}$ and $P_{\text {meas }}$ were equal. Thus, the flow rate was set in proportion to the difference between the reference and measured values.

Flow rate $=$ constant $\left(\mathbf{P}_{\text {ref }}-\mathbf{P}_{\text {meas }}\right)$.

The constant, equaling the gain of the feedback loop, determined the dynamical response of the system. It was chosen large enough to assure that the response time of the feedback loop (less than $1 \mathrm{~s}$ ) was much faster than the intrinsic response time of the artery and small enough to prevent overshoot of the arterial pressure above the reference pressure (the pump could not apply suction). The reference pressure was changed at fixed intervals (every $5 \mathrm{~min}$ ), and the volume of fluid pumped (net displacement of the syringe) was stored periodically (every $3 \mathrm{~s}$ ) as a function of time in the computer. To start the measurements, the segment of isolated artery was submitted to atmospheric pressure for $5 \mathrm{~min}$ and then to a pressure step of $25 \mathrm{mmHg}$. During the first $30-45 \mathrm{~s}$ the inflow was rapid, and then became constant with time. The initial rapid increase in volume with pressure was assumed to result from the viscoelastic behavior of the tissue and the relaxation of the vascular smooth muscle. The later constant flow into the carotid artery after the initial increase in arterial volume was attributed to the
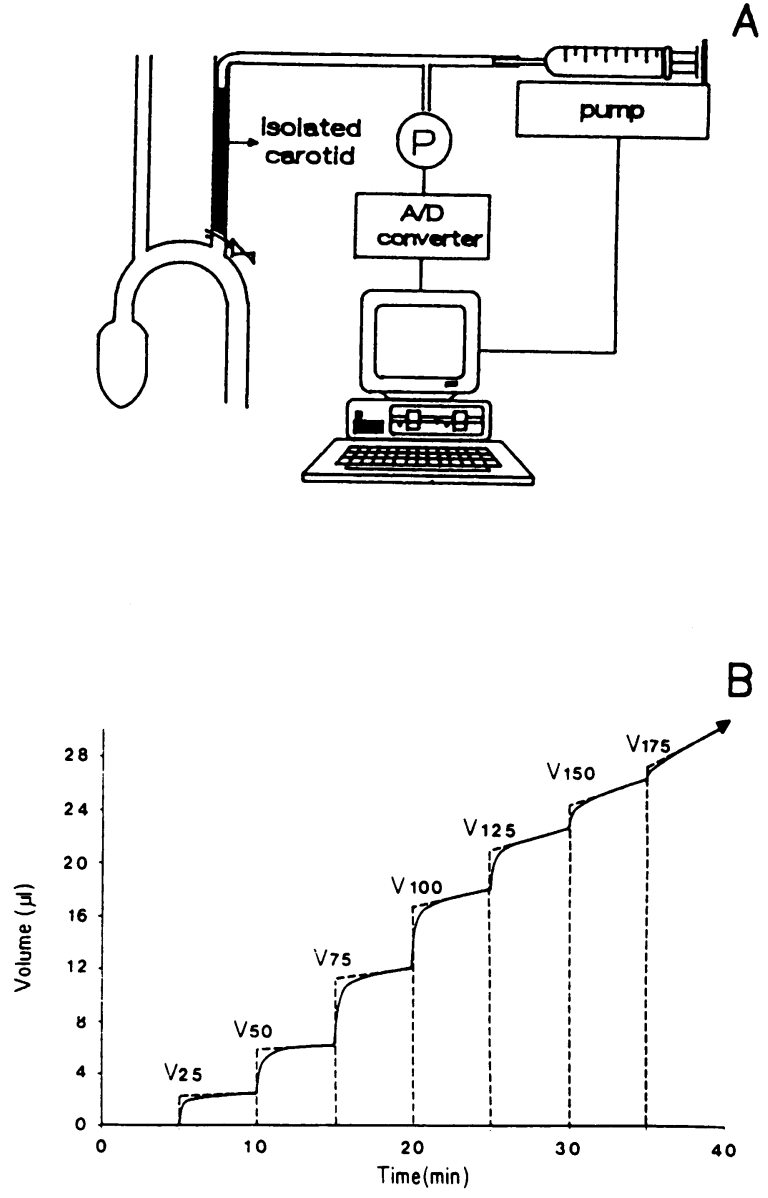

Figure 1. (A) Schematic representation of the experimental set-up permitting us to measure the volume-pressure relation in the in situ isolated carotid artery. $(B)$ Pressure-volume relation in the carotid artery. This figure illustrates the pressure-volume relation in the carotid artery, including the effect of fluid filtration across the arterial wall. V25, V50 are the successive volumes, free of viscoelastic and filtration effects, induced by the pressure steps.

fluid filtration across the vascular wall. An estimate of the static increase in volume, free of fluid filtration, was obtained by extrapolating the linear portion of the volume curve to the time when the pressure step was applied (Fig. $1 b$ ). The slope of the late linear portion of the injected volume vs. time relationship represents the filtration rate of fluid across the wall of the carotid artery. These measurements were repeated for pressures ranging from 25 to $200 \mathrm{mmHg}$ in steps of 25 $\mathrm{mmHg}$. The static compliance of the isolated segment of carotid artery was automatically calculated for each level of pressure, as the quotient of the extrapolated volume increase and the pressure step imposed ( 25 $\mathrm{mmHg}$ ). In preliminary experiments we have verified that the carotid compliance values were not different when measured for pressure increment (from 25 to $200 \mathrm{mmHg}$ ) or for pressure decrement (from 200 to $25 \mathrm{mmHg}$ ).

After termination of the measurements under control conditions, the carotid clamp was removed, and the artery was rinsed with the Tyrode's solution. Subsequently, the clamp was replaced and the artery filled with a saline solution containing $100 \mathrm{mg} /$ liter potassium cyanide $(\mathrm{KCN})$. The $\mathrm{KCN}$ solution was maintained in the vessel for $30 \mathrm{~min}$, to poison the smooth muscle mass completely. Afterwards the vessel was rinsed and refilled with the modified Tyrode's solution, and compliance measurements were performed again to determine carotid compliance in the fully relaxed state, depending only on passive elastic properties. Preliminary experiments, in which carotid compliance measure- 
ments were performed with a 1-h interval, showed that the duration of total protocol did not affect static compliance values.

Fluid filtration rate. The slope of the late linear portion of the injected volume vs. time relationship represents the filtration rate of fluid through the wall of the carotid artery. This filtration rate was calculated for each pressure step imposed, and expressed as $\mathrm{nl} / \mathrm{s}$ per $\mathrm{mm}$ of artery.

Left ventricular wt/body wt ratio. After termination of the experiment, when rats were still anesthetized, the heart was excised, and, after removal of both atria and the right ventricle, left ventricular weight was determined and corrected for the body weight of the animal.

Statistical analysis. All results are expressed as mean \pm SD. Differences between groups were analyzed using ANOVA. Carotid compliance curves were compared using one-way ANOVA for each pressure level. Fluid filtration curves were compared using logistic regression. Statistical significance was assumed at the $P<0.05$ level.

\section{Results}

General characteristics. Body wt were comparable in both groups and relatively low ( $188 \pm 33 \mathrm{~g}$ in untreated diabetic rats vs. $188 \pm 23$ in the aminoguanidine-treated group) as a result of the prolonged hyperglycemia. Blood glucose levels were equally elevated in both groups $(22.8 \pm 2.3 \mathrm{mmol} /$ liter in untreated diabetic rats vs. $23.5 \pm 2.9 \mathrm{mmol} /$ liter in aminoguanidine-treated rats). The left ventricular wt/body wt ratio was slightly but significantly lower in aminoguanidine-treated rats compared to untreated rats $(3.1 \pm 0.2 \mathrm{mg} / \mathrm{g}$ body wt vs. $3.5 \pm 0.4$ $\mathrm{mg} / \mathrm{g}$ body wt, $P<0.05$ ).

Hemodynamic parameters. Systolic, diastolic, and mean arterial blood pressure, determined under anesthetized openchest conditions, were slightly but not significantly higher $(P$ $=0.13$ ) in the aminoguanidine-treated group (Fig. 2). Pulse pressure, an indirect parameter of arterial elasticity was $15 \%$ lower in aminoguanidine-treated animals $(P<0.05$, Fig. 2$)$. Cardiac output was comparable in both groups $(71 \pm 12 \mathrm{ml} /$ min in diabetic rats vs. $77 \pm 11$ in aminoguanidine-treated diabetic rats). Aminoguanidine treatment also resulted in a $40 \%$ lower characteristic aortic input impedance in diabetic rats $(P$ $<0.01$, Fig. $3 a$ ). The $\dot{\mathrm{W}}_{\mathrm{o}} / \dot{\mathrm{W}}_{\mathrm{t}}$ ratio, reflecting the relative contribution of oscillatory power to total ventricular power output and therefore the efficacy of coupling between the left ventricle and the arterial system, was also markedly lower following aminoguanidine treatment $(-35 \%, P<0.05$, Fig. $3 b)$.

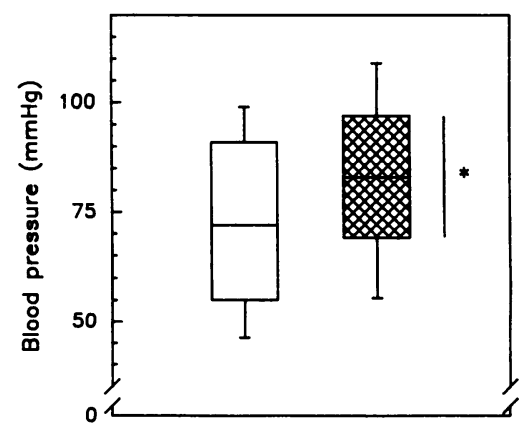

Figure 2. Systolic, diastolic, and mean arterial blood pressure in anesthetized, open-chest, diabetic, and aminoguanidine-treated diabetic rats. The upper level of the box represents systolic blood pressure (SBP), the middle line mean arterial blood pressure (MAP), and the lower level of the box diastolic blood

pressure (DBP). The height of the box reflects the pulse pressure (PP). SBP, MAP, and DBP were slightly but not significantly higher $(P=0.13)$ in aminoguanidine-treated diabetic rats (hatched box) compared to diabetic rats (blank box). PP was significantly lower in aminoguanidine-treated rats $\left({ }^{*} P<0.05\right)$.
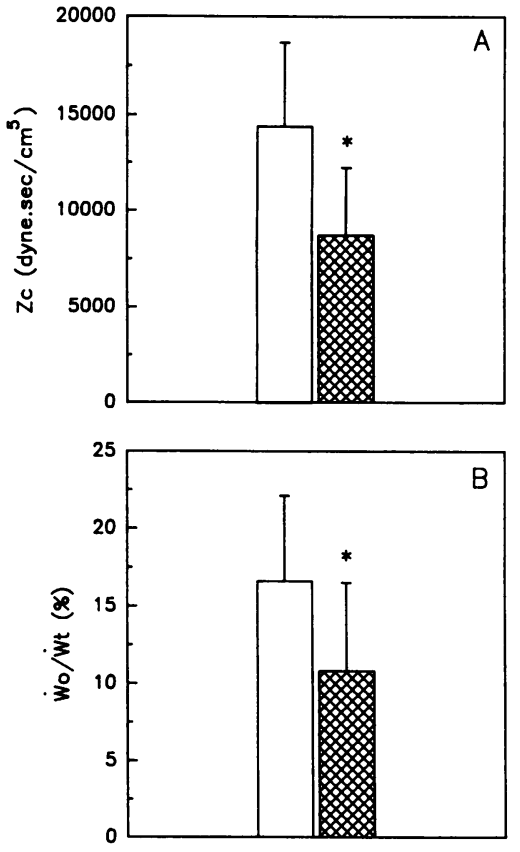

Figure 3. Characteristic aortic input impedance, $\mathrm{Zc}$ (graph $A$ ), and the left ventricular oscillatory power output/total power output ratio, $\dot{\mathrm{W}}_{0} / \dot{\mathrm{W}}_{\mathrm{t}}($ graph $B)$. Both parameters were determined from Fourier series of aortic pressure and flow. $\mathrm{Zc}$ and $\dot{\mathrm{W}}_{0} /$ $\dot{\mathrm{W}}_{\mathrm{t}}$ were equally reduced in aminoguanidine-treated rats (hatched bars) compared to diabetic rats (blank bars) $\left({ }^{*} P<0.01\right.$ and $P<0.05$, respectively).

Carotid artery compliance. Static compliance of the carotid artery under basal conditions and after incubation of the vessel with $\mathrm{KCN}$ is depicted in Fig. $4 a$ and $b$. Carotid artery compliance is pressure dependent and was markedly higher in aminoguanidine-treated diabetic rats compared to untreated rats in
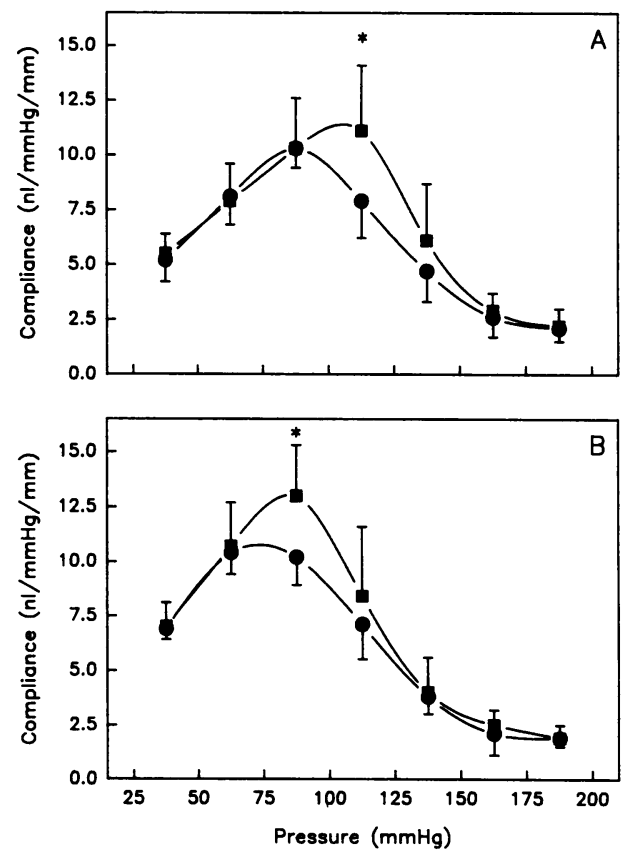

Figure 4. Carotid artery compliance. Static compliance of the in situ isolated carotid artery vs. pressure. Graph $A$ reflects compliance under control conditions while graph $B$ reflects compliance after incubation with $\mathrm{KCN}$ to abolish vascular tone. Carotid compliance was significantly higher in aminoguanidine-treated rats (closed squares, $P$ $<0.01$ ) compared to diabetic rats (closed circles) in the range of 100 to $125 \mathrm{mmHg}$, matching the operating pressure in these rats. After incubation with $\mathrm{KCN}$, both curves shifted to the left, but carotid compliance remained higher in aminoguanidine-treated rats $(P<$ $0.01)$. 


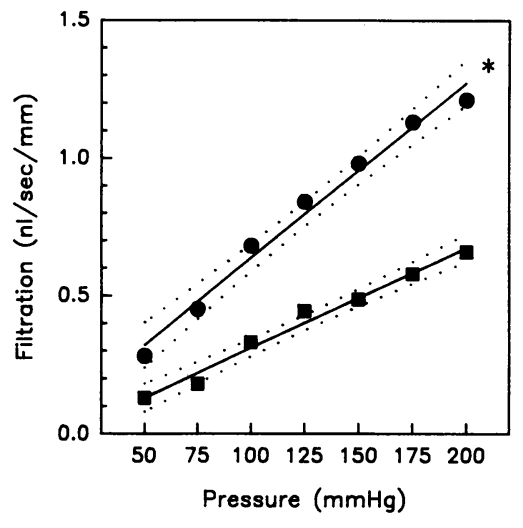

tion across the carotid arterial wall of aminoguanidine-treated rats was significantly lower compared to untreated rats $(P<0.05)$.

the range of $100-125 \mathrm{mmHg}(+39 \%, P<0.01)$. Incubation of the carotid artery with $\mathrm{KCN}$, resulting in maximal dilation of the vessel, shifted both curves to the left. Under dilated conditions, carotid compliance was clearly elevated in the aminoguanidine group compared to the untreated diabetic group in the range of $75-100 \mathrm{mmHg}(+27 \%, P<0.01)$.

Fluid filtration rate. Fluid filtration showed a linear relation with pressure and was significantly lower over the complete pressure range in aminoguanidine-treated rats (Fig. 5).

\section{Discussion}

In this study we investigated whether treatment with aminoguanidine, a drug known to inhibit AGE accumulation on collagen, influences mechanical properties of large compliance arteries in diabetic rats. This approach allows us to quantify arterial wall properties in rats that are comparable in body wt. The large differences in body wt that develop between control and streptozotocin diabetic rats in long-term studies $(10,11)$ would make it difficult to interpret parameters that are influenced by vascular dimensions, such as characteristic aortic input impedance and carotid artery compliance.

Aminoguanidine treatment resulted in a slight increase in mean arterial pressure. Cardiac output was almost comparable between the two groups, suggesting that an increased total peripheral resistance might contribute to this small rise in blood pressure. Corbett et al. (12) have shown that aminoguanidine is able to inhibit nitric oxide formation and thus can increase systemic blood pressure, although its effects are much weaker than those of established nitric oxide synthase inhibitors such as $N^{\mathrm{G}}$-monomethyl-L-arginine (L-NMMA). However, in both groups of rats, mean arterial pressure, determined under anesthetized open-chest conditions, was relatively low. This is in agreement with the results of Litwin et al. (13), who showed that thoracotomy lowered mean arterial pressure about $25 \%$ in diabetic rats, in contrast to a $10 \%$ decrease in control rats. Indeed, mean arterial pressure in diabetic rats of this strain in the conscious state ranges in our laboratory from 100 to 120 $\mathrm{mmHg}$.

In comparison with untreated rats, carotid compliance in rats treated with aminoguanidine was markedly higher in the pressure range of $100-125 \mathrm{mmHg}$, the pressure that matches the actual blood pressure in these rats. The increase in carotid compliance was observed both under basal conditions and after abolition of vascular tone by KCN. This makes it unlikely that carotid compliance is solely enhanced through a modulation of vascular tone induced by aminoguanidine treatment. Bucala et al. (14) have shown that AGEs are able to quench nitric oxide and in this way interfere with its vasoactive properties. Aminoguanidine treatment could thus, by inhibiting the formation of AGEs on vascular wall proteins, prevent this quenching, resulting in a relative dilation of vessels and probably a larger arterial compliance in the aminoguanidine-treated group, although this would be contradictory to the inhibiting effects on NO-formation described above. Nevertheless, after maximal dilation induced by $\mathrm{KCN}$ incubation, carotid compliance was still substantially higher in the aminoguanidinetreated group, indicating that passive arterial wall properties are changed markedly by aminoguanidine treatment.

Characteristic aortic input impedance constitutes another index of vascular elasticity that is determined independently of carotid compliance. The observed decrease in characteristic aortic input impedance after aminoguanidine treatment supports our hypothesis that aminoguanidine treatment, by preventing AGE accumulation on collagen, can increase vascular elasticity in diabetes. The decreased ratio of left ventricular oscillatory power output to total ventricular power output indicates that less energy was lost in pulsatile components, as was reflected also in a lower pulse pressure in the aminoguanidinetreated group. Moreover, left ventricular wt/body wt ratio was also decreased, suggesting a diminished left ventricular load, despite the slight increase in mean arterial pressure.

Alterations in arterial elasticity have gained much attention in hypertension research. Many studies have been performed regarding mechanical arterial wall properties, such as arterial compliance, in both clinical and experimental hypertension (15-17). The important consequences of alterations in vascular elasticity on cardiovascular performance have been shown unequivocally. A decrease in arterial compliance by itself does not affect mean arterial pressure but it amplifies the pulse pressure. Increased pulse pressure is an independent risk factor for cardiovascular complications (18). Besides, aortic compliance and characteristic aortic input impedance are strongly related to cardiac mass $(19,20)$. Although similar studies have not yet been performed in diabetic patients, changes in arterial elasticity are likely to have important (negative) effects on cardiovascular functioning in diabetes as well.

In addition to the compliance measurements of the carotid artery, we were able to quantify fluid filtration across the carotid wall, and we showed that aminoguanidine treatment resulted in a marked decrease of fluid filtration. Increased vascular permeability is a well-established feature of diabetic angiopathy and has been shown in both experimental $(21,22)$ and clinical $(23,24)$ studies. Whereas fluid filtration represents hydraulic conductivity of the vascular wall and is not exactly analogous to vascular permeability, measurement of fluid filtration might give an indication of vascular permeability to macromolecules as well. By which mechanisms aminoguanidine might reduce vascular permeability is not well known yet. In experimental diabetes, however, aminoguanidine was shown to diminish albuminuria $(25,26)$, a reflection of increased glomerular permeability, possibly by preventing the decreased binding of heparan-sulphate to AGE-modified basement membrane components $(27,28)$.

Summarizing our results, we provided evidence that treatment of diabetic rats with aminoguanidine significantly in- 
creased vascular elasticity, indicating that AGE accumulation on vascular wall collagen markedly affects mechanical arterial wall properties. Moreover, aminoguanidine treatment reduced fluid filtration across the carotid arterial wall, which might be a reflection of normalized vascular permeability. Together with its already published beneficial effects (14), this suggests that aminoguanidine can protect the cardiovascular system in (experimental) diabetes.

\section{References}

1. Monnier, V. M., R. R. Kohn, and A. Cerami. 1984. Accelerated age-related browning of human collagen in diabetes mellitus. Proc. Natl. Acad. Sci. USA. 81:583-587.

2. Schnider, S. L., and R. R. Kohn. 1980. Glycosylation of human collagen in aging and diabetes mellitus. J. Clin. Invest. 66:1179-1181.

3. Yue, D. K., S. McLennan, L. Delbridge, D. J. Handelsman, T. Reeve, and J. R. Turtle. 1983. The thermal stability of collagen in diabetic rats: correlation with severity of diabetes and non-enzymatic glycosylation. Diabetologia. 24:282285.

4. Brownlee, M., H. Vlassara, A. Kooney, P. Ulrich, and A. Cerami. 1986 Aminoguanidine prevents diabetes-induced arterial wall protein cross-linking Science (Wash. DC). 232:1629-1632.

5. Kumari, I., S. Umar, V. Bansal, and M. K. Sahib. 1991. Inhibition of diabetes associated complications by nucleophilic compounds. Diabetes 40:1079-1084

6. Edelstein, D., and M. Brownlee. 1992. Mechanistic studies of advanced glycosylation endproduct inhibition by aminoguanidine. Diabetes. 41:26-29.

7. Benessiano, J., B. I. Lévy, and J. B. Michel. 1985. Instantaneous aortic blood flow measurements with range gated Doppler flowmeter in anesthetized rat. J. Pharmacol. Methods 14:99-110.

8. Milnor, W. R. 1989. Cardiac dynamics. In Hemodynamics. N. Collins, editor. Williams \& Wilkins, Baltimore. 275-293.

9. Morrisson, A. D., L. Berwick, L. Orci, and A. I. Winegrad. 1976. Morphology and metabolism of an aortic intima-media preparation in which an intact endothelium is preserved. J. Clin. Invest. 57:650-660.

10. Suarez, G., R. Rajaram, K. C. Bhuyan, A. L. Oronsky, and J. A. Goidi. 1988. Administration of an aldose reductase inhibitor reduces a decrease of collagen fluorescence in diabetic rats. J. Clin. Invest. 82:624-627.

11. Lash, J. M., and H. G. Bohlen. 1991. Structural and functional origins of suppressed acetylcholine vasodilation in diabetic rat intestinal arterioles. Circ. Res. 69:1259-1268.

12. Corbett, J. A., R. G. Tilton, K. Chang, K. S. Hasan, Y. Ido, J. L. Wang, M. A. Sweetland, J. R. Lancaster, J. R. Williamson, and M. L. McDaniel. 1992. Aminoguanidine, a novel inhibitor of nitric oxide formation, prevents diabetic vascular dysfunction. Diabetes. 41:552-556.
13. Litwin, S. E., T. E. Raya, S. Daugherty, and S. Goldman. 1991. Peripheral control of cardiac output in diabetic rats. Am. J. Physiol. 30:H836-H842.

14. Bucala, R., K. J. Tracey, and A. Cerami. 1991. Advanced glycosylation products quench nitric oxide and mediate defective endothelium-dependent vasodilatation in experimental diabetes. J. Clin. Invest. 87:432-438.

15. Safar, M. E., and G. M. London. 1987. Arterial and venous compliance in sustained essential hypertension. Hypertension (Dallas). 10:133-139.

16. Lévy, B. I., J. Benessiano, P. Poitevin, and M. E. Safar. 1990. Endothelium-dependent mechanical properties of the carotid artery in WKY and SHR Role of angiotensin converting enzyme inhibition. Circ. Res. 66:321-328.

17. Lévy, B. I., P. Poitevin, and M. E. Safar. 1991. Effects of alphal-blockade on arterial compliance in normotensive and hypertensive rats. Hypertension (Dallas). 17:534-540.

18. Darne, B., X. Girerd, M. E. Safar, F. Cambien, and L. Guize. 1989 Pulsatile versus steady component of blood pressure: a cross-sectional analysis and a prospective analysis on cardiovascular mortality. Hypertension (Dallas). 13:392-400.

19. Isnard, R. N., B. M. Pannier, S. Laurent, G. M. London, B. Diebold, and M. E. Safar. 1989. Pulsatile diameter and elastic modulus of the aortic arch in essential hypertension: a non-invasive study. J. Am. Coll. Cardiol. 13:399-405.

20. Lévy, B. I., D. Babalis, P. Lacolley, P. Poitevin, and M. E. Safar. 1988. Cardiac hypertrophy and characteristic impedance in spontaneously hypertensive rats. J. Hypertens. 6 (Suppl. 4):S110-S111.

21. Williamson, J. R., K. Chang, R. G. Tilton, C. Prater, J. R. Jeffrey, C. Weigel, W. R. Sherman, D. M. Eades, and C. Kilo. 1987. Increased vascular permeability in spontaneously diabetic $\mathrm{BB} / \mathrm{W}$ rats and in rats with mild versus severe streptozotocin-induced diabetes. Prevention by aldose reductase inhibitors and castration. Diabetes. 36:813-821.

22. Pugliese, G., R. G. Tilton, A. Speedy, E. Santarelli, D. Eades, M. A. Province, C. Kilo, W. R. Sherman, and J. R. Williamson. 1990. Modulation of hemodynamic and vascular filtration changes in diabetic rats by dietary myo-inositol. Diabetes. 39:312-322.

23. Bollinger, A., J. Frey, K. Jaeger, J. Furrer, J. Seglias, and W. Siegenthaler. 1982. Patterns of diffusion through skin capillaries in patients with long-term diabetes. N. Engl. J. Med. 307:1305-1310.

24. Feldt-Rasmussen, B. 1986. Increased transcapillary escape rate of albu$\min$ in Type 1 (insulin-dependent) diabetic patients with microalbuminuria. Diabetologia. 29:282-286.

25. Edelstein, D., and M. Brownlee. 1992. Aminoguanidine ameliorates albuminuria in diabetic hypertensive rats. Diabetologia. 35:96-97.

26. Soulis-Liparota, T., M. Cooper, D. Papazoglou, B. Clarke, and J. Jerums. 1991. Retardation by aminoguanidine of development of albuminuria, mesangial expansion, and tissue fluorescence in streptozotocin-induced diabetic rat. Diabetes. 40:1328-1334.

27. Charonis, A. S., L. A. Reger, J. E. Dege, K. Kouzii-Koliakos, L. T. Furcht, R. M. Wohlhueter, and E. T. Tsilibary. 1990. Laminin alterations after in vitro non-enzymatic glycosylation. Diabetes. 39:807-814

28. Brownlee, M. 1992. Glycation products and the pathogenesis of diabetic complications. Diabetes Care. 15:1835-1843. 